\title{
IMPACTO DE LA PANDEMIA COVID-19 EN EL PERSONAL MÉDICO DE LOS SERVICIOS DE CIRUGIA GENERAL DEL HOSPITAL DR. MIGUEL PÉREZ CARREÑO. ESTUDIO DE COHORTE
}

\author{
SILVIA PIÑANGO' \\ LUIS LEVEL ${ }^{2}$ (D) \\ CRISTINA INCHAUSTI ${ }^{3}$
}

\begin{abstract}
IMPACT OF THE COVID-19 PANDEMIC ON THE MEDICAL STAFF OF GENERAL SURGERY SERVICES AT DR. MIGUEL PÉREZ CARREÑO HOSPITAL. COHORT STUDY
\end{abstract}

\section{RESUMEN}

Las patologías quirúrgicas continúan siendo motivo de consulta habitual en los servicios de emergencia de nuestros hospitales, requiriendo una rápida evaluación y resolución oportuna aún en tiempos de pandemia, siendo muy probable que pacientes asintomáticos, con sospecha o confirmación de infección por COVID-19 ameriten una intervención quirúrgica. Objetivo: Analizar la incidencia de casos de COVID-19 y evaluar su correlación con el uso de equipos de protección personal en los médicos residentes y adjuntos de los servicios de cirugía general del Hospital Dr. Miguel Pérez Carreño. Métodos: Se realizó una investigación de tipo observacional, descriptiva y transversal. La muestra estuvo constituida por 32 médicos quienes respondieron anónimamente una encuesta electrónica realizada por el comité académico del Posgrado de Cirugía General. Resultados: El porcentaje global de participación fue 50,79\%. Resultaron positivos para la prueba Reacción en Cadena de Polimerasa el 42\% de los residentes y $9 \%$ de los médicos especialistas. El lugar más frecuente de probable contacto fue el hospital en $90 \%$ de los casos. El 50\% de los médicos identificaron a un personal de salud como su fuente de contagio. Se reportó uso constante de equipo de protección personal y gel alcoholado en $47 \%$ y $72 \%$ de los casos respectivamente. Conclusión: El aumento de casos de COVID-19 en la población general y en el personal de salud debe alertarnos acerca de la necesidad de tomar medidas estrictas de protección en forma precoz orientadas a disminuir la incidencia de casos y por ende la morbilidad y la mortalidad asociada a la infección por el nuevo coronavirus.

Palabras clave: Pandemia, coronavirus, COVID-19, cirugía general, residentes, adjuntos, personal de salud, equipos de protección personal

1. Cirujano General. Coordinador del Postgrado de Cirugía General de la Universidad Central de Venezuela. Hospital Dr. Miguel Pérez Carreño. Caracas - Venezuela.

2. Cirujano General. Jefe de Servicio de Cirugía I. Director del Postgrado de Cirugía General de la Universidad Central de Venezuela. Hospital Dr. Miguel Pérez Carreño. Caracas Venezuela. Correo-e: levelluis@yahoo.com

3. Residente de 3er año del Servicio de Cirugía I. Hospital Dr. Miguel Pérez Carreño. Caracas - Venezuela.

\begin{abstract}
Surgical pathologies continue to be a common reason for consultation in the emergency services of our hospitals, requiring a rapid evaluation and timely resolution even in times of pandemic, and it is highly probable that asymptomatic patients, with suspected or confirmed COVID-19 infection, merit a surgical intervention. Objective: To analyze the incidence of COVID-19 cases and evaluate its correlation with the use of personal protective equipment (PPE) in resident and staff surgeons of General Surgery services at Dr. Miguel Pérez Carreño Hospital. Methods: An observational, descriptive and cross-sectional research was carried out. The sample consisted of 32 physicians who anonymously responded to an electronic survey conducted by the academic committee of the General Surgery residency program. Results: The overall percentage of participation was $50.79 \%$. 42\% of residents and $9 \%$ of specialist physicians were positive for the Polymerase Chain Reaction test. The most frequent place of probable contact was the hospital in $90 \%$ of the cases. 50\% of the doctors identified a health personnel as their source of infection. Constant use of PPE and alcohol gel was reported in $47 \%$ and $72 \%$ of the cases, respectively. Conclusion: The increase in COVID-19 cases in the general population and in health personnel should alert us to the need to take stricter protection measures early in order to reduce the incidence of cases and, therefore, the morbidity and mortality associated with infection with the new coronavirus.
\end{abstract}

Key words: Pandemic, coronavirus, COVID-19. general surgery, residents, staff surgeons, health personnel, personal protection equipment. 


\section{INTRODUCCIÓN}

La enfermedad viral infecto-contagiosa por coronavirus 2019 (COVID-19) fue detectada por primera vez en la ciudad de Wuhan (China) en diciembre de 2019 '1!, propagándose a un ritmo exponencial por todo el mundo hasta ser declarada pandemia por la Organización Mundial de la Salud el día 11 de marzo de 2020 (2). El primer caso de infección por SARS-CoV-2 fue detectado en Caracas (Venezuela) el 13 de marzo de 2020. El 14 de marzo el gobierno nacional decretó estado de alarma en todo el territorio nacional anunciando las nuevas medidas para evitar la propagación del virus en la población. ${ }^{|3|}$

En poco tiempo se han redistribuido los recursos y la organización de los centros hospitalarios, especialmente en los servicios de urgencias y unidades de cuidados intensivos (UCI), adaptándose a la situación y organizando la asistencia de los pacientes COVID-19 en función a las necesidades diarias. ${ }^{(4)}$

Las patologías quirúrgicas y por traumatismos continúan siendo motivo de consulta habitual en los sistemas de emergencia, requiriendo rápida evaluación y resolución oportuna aun en tiempos de pandemia, siendo muy probable que pacientes con sospecha o confirmación de infección por COVID-19 ameriten una intervención quirúrgica. En estos casos se deben tener protocolos y equipos de protección personal (EPP) para el cuidado especial del personal de salud y de los pacientes que se asisten, muchos de los cuales ingresan en estado crítico y con necesidad de intervención quirúrgica inmediata que no permite la demora en su resolución. ${ }^{(5)}$

En este estudio, se realiza un análisis descriptivo sobre el impacto que ha tenido la pandemia por SARS-CoV-2 en los servicios de cirugía general del Hospital Dr. Miguel Pérez Carreño en los primeros 6 meses desde la aparición del virus en nuestra ciudad.

En razón de la afectación de esta enfermedad también a pacientes quirúrgicos y al equipo de salud, el objetivo es analizar la incidencia de casos en los médicos residentes y adjuntos de los cuatro servicios de cirugía del hospital, así como estudiar las posibles causas de contagio y evaluar el cumplimiento de las medidas de protección por el personal adscrito.

\section{MÉTODOS}

Se desarrolló un estudio observacional, descriptivo y transversal, enmarcado en una investigación epidemiológica, de campo, no experimental. La población estuvo constituida por todos los médicos residentes y adjuntos de los cuatro servicios de cirugía general del hospital Dr. Miguel Pérez Carreño quienes respondieron una encuesta electrónica proporcionada por el comité académico del Posgrado de Cirugía General entre los meses de agosto y septiembre de 2020. Se incluyeron todos los residentes de los tres años de posgrado de los cuatro servicios, así como los médicos especialistas adscritos a los cuatro servicios de cirugía general del hospital Dr. Miguel Pérez Carreño.
Se aplicó un cuestionario diseñado en línea en un formulario de Google, elaborado por miembros del Comité Académico del Posgrado de Cirugía General. El mismo fue enviado posteriormente al comité conformado por los jefes de los cuatro servicios, el coordinador académico del posgrado, un representante de los adjuntos y un representante estudiantil, para su aprobación y sugerencias, las cuales fueron incorporadas en la versión final. La encuesta fue anónima, se distribuyó vía correo electrónico y vía telefónica por la aplicación WhatsApp@. El cuestionario incluía 26 preguntas con cuatro opciones y dos preguntas abiertas sobre preocupaciones y sugerencias que se incluyeron en el análisis cualitativo del estudio. Las preguntas eran de respuesta obligatoria para poder concluir el envío de los participantes. Enlace para visualizar formulario.

El análisis de los resultados se realizó mediante descripción porcentual de las variables.

\section{RESULTADOS}

El estudio registró la participación de 32 médicos, con todos los datos del formulario completados, lo que representa el 50,8\% del personal de residentes y adjuntos adscritos a los cuatro servicios de cirugía general del Hospital Dr. Miguel Pérez Carreño. Los restantes 31 médicos no contestaron el formulario (Tabla 1).

\begin{tabular}{|c|c|c|c|}
\hline $\begin{array}{c}\text { Nivel } \\
\text { académico }\end{array}$ & Total & Participantes & $\begin{array}{l}\text { Participación } \\
\text { (\%) }\end{array}$ \\
\hline Residentes & 36 & 21 & 58,33 \\
\hline Nivel I & 14 & 6 & 42,85 \\
\hline Nivel II & 9 & 4 & 44,44 \\
\hline Nivel III & 13 & 11 & 84,61 \\
\hline Adjuntos & 27 & 11 & 40,74 \\
\hline Total & 63 & 32 & 50,79 \\
\hline
\end{tabular}

En lo que respecta al nivel académico se registró un 65,8\% de médicos residentes de posgrado de cirugía y el 34,4\% médicos adjuntos. La mayor participación de los residentes fue de los residentes de tercer año con 34,4\% seguido por los estudiantes de primer año con 18,8\% y con un 12,5\% los residentes de segundo aก̃o.

El tipo de método de transporte más utilizado fue el carro propio 65,6\%, seguido por transporte público 28, 1\%, taxi 12,5\%, caminando 6,3\%, carro con otro residente 6,3\% y otro tipo de transporte $9,4 \%$.

El 28,1\% de los participantes en el estudio refirió haber asistido durante la pandemia a alguna reunión social.

El 65,8\% de los encuestados refirió haber participado en la cirugía o en la atención pre o posoperatoria de pacientes con sospecha o confirmación de COVID-19.(Gráfico 1-a) El 31,3\% de los participantes asistió a guardias en el área de aislamiento de pacientes COVID-19 del hospital. (Gráfico 1-D). 


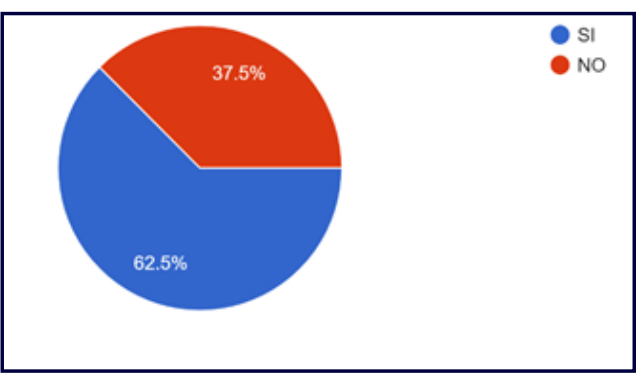

a) Atención o participación en cirugías de paciente con sintomatología respiratoria

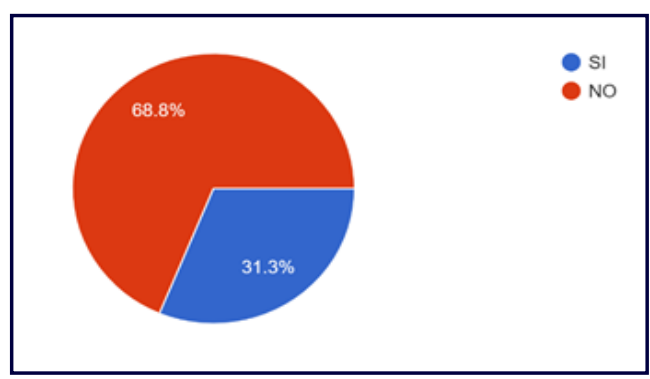

b) Asistencia al área de aislamiento COVID del hospital

El promedio de días entre el posible contacto con un paciente contagiado e inicio de síntomas fue de 4,25 días. De los participantes que ameritaron tratamiento todos refirieron haber comprado su tratamiento, ningún participante refirió haber recibido tratamiento del hospital o alguna entidad.

\section{Gráfico 1. Situaciones de riesgo en la atención médica}

El 50\% de los médicos sintomáticos refirió haberse reintegrado a sus actividades laborales,

De los 32 participantes el 62,5\% refirió no haber tenido síntomas, 31,3\% haber tenido síntomas leves y 6,3\% síntomas moderados.

Del total de participantes se realizó toma de muestra de hisopado nasofaríngeo al 56,4\% con resultado de la reacción en cadena de polimerasa positivo en el 31,3\%, negativo en el $18,8 \%$ y el 6,3\% en espera de resultados para el momento del estudio. (Gráfico 2)

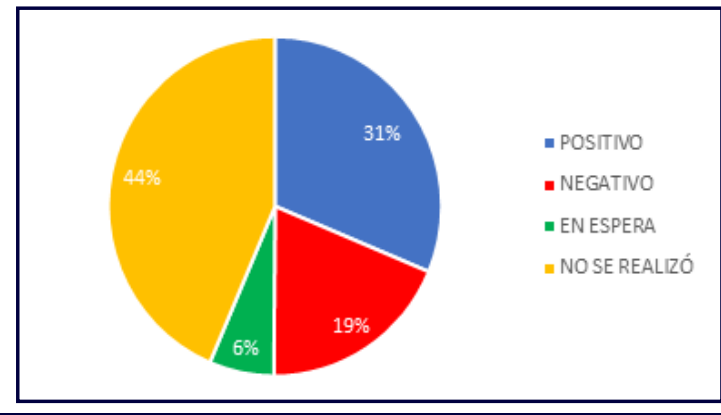

Gráfico 2. Resultado de PCR para COVID 19

El 90\% de los médicos contagiados refiere conocer el origen de su infección. El 90\% refirió haber tenido contacto con una persona sintomática, el 90\% refirió posible contagio en el hospital y el 10\% en un centro privado de salud.

El 50\% de la población estudiada aseguró que la fuente de su infección fue un paciente positivo para COVID-19 mientras que el otro el 50\% refirió que la fuente fue contacto con personal de salud. (Gráfico 3)

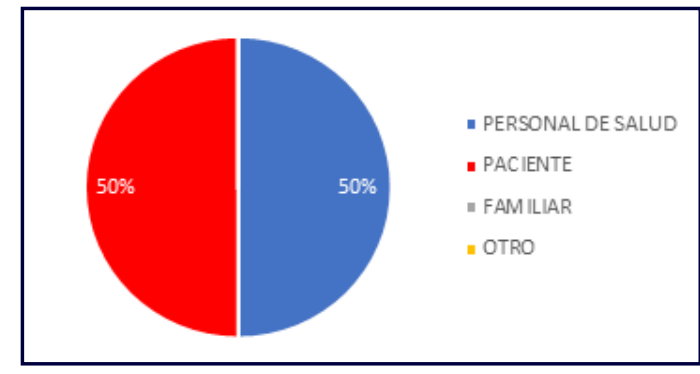

Gráfico 3. Persona quien considera como su fuente de contagio mientras que la otra mitad refirió encontrarse en aislamiento domiciliario para el momento del estudio.

En lo que respecta a los métodos de protección, el 83,1\% refirió conocer y aplicar el procedimiento para colocar y retirar el EPP. El 46,9\% refirió usar siempre EPP, 28, 1\% algunas veces, 9,4\% casi nunca y 15,6\% nunca. (Gráfico 4)

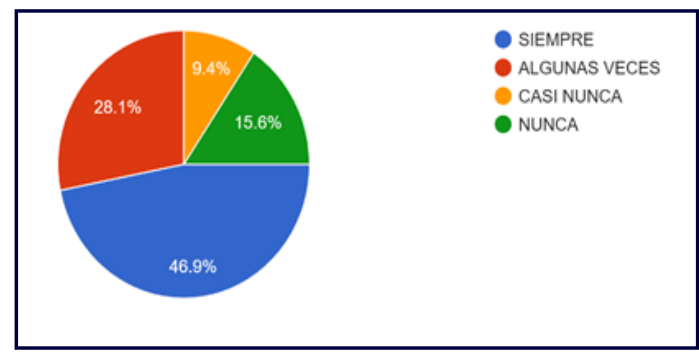

Gráfico 4. Uso de equipo de protección personal

Del total de participantes el 71,9\% refirió uso de gel alcoholado siempre, 25\% algunas veces y el 3,1\% casi nunca.

En el nivel básico de atención refirieron el uso de gel alcoholado 87,5\%, gorro 87,5\%, guantes 68,8\%, mascarilla quirúrgica 78, 1\%, N95 31,3\%, lentes de protección 21,9\%, careta 71,9\%, braga 3,1\%, botas 28, 1\%. (Gráfico 5-a)

En el nivel reforzado de atención refirieron el uso de gel alcoholado 87,5\%, gorro 90,6\%, guantes 84,4\%, mascarilla quirúrgica 68,8\%, N95 50\%, lentes de protección 31,3\%, careta 87,5\%, braga 21,9\%, botas 50\%. (Gráfico 5-b)

En los procedimientos generadores de aerosoles refirieron el uso de gel alcoholado 87,5\%, gorro 93,8\%, guantes 90,6\%, mascarilla quirúrgica 78,1\%, N95 65,5\%, lentes de protección 43,8\%, careta 93,8\%, braga 34,4\%, botas 68,8\%. (Gráfico 5-c)

Enlace para visualizar los resultados

\section{DISCUSIÓN}

En el presente estudio se registró una participación de un número importante del personal médico adscrito a los cuatro servicios de cirugía del hospital Dr. Miguel Pérez Carreño siendo el mayor porcentaje el de médicos residentes. La disponibilidad 


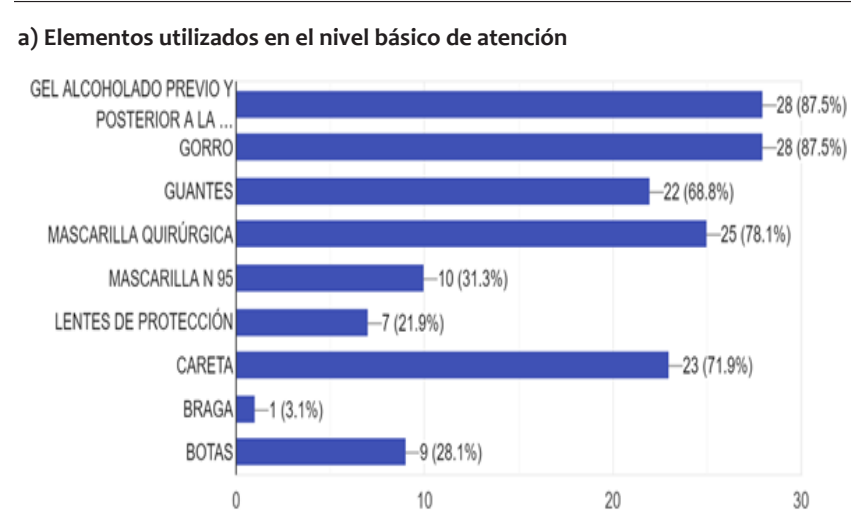

b) Elementos utilizados en el nivel reforzado de atención

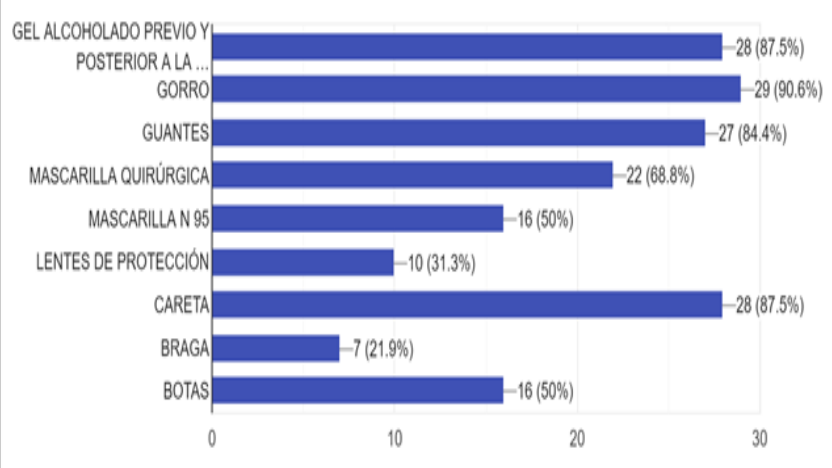

c) Elementos utilizados en procedimientos generadores de aerosoles

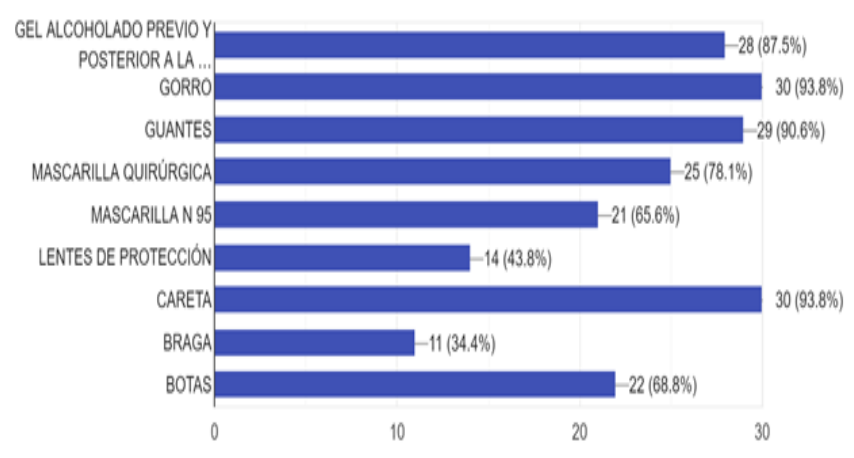

Gráfico 5. Elementos utilizados según el nivel de atención

de dispositivo inteligente o el acceso a internet constituyó una fuente de sesgo para la participación en el protocolo.

A pesar de que el método de transporte más utilizado fue el carro propio, un porcentaje importante del personal médico de cirugía utilizó otros tipos de transporte los cuales pudieron por sus características aumentar el riesgo de contagio y de transmisión de la enfermedad.

Al inicio de la pandemia nuestro hospital destinó un área específica para la atención de pacientes con clínica respiratoria y hospitalización de pacientes con insuficiencia respiratoria de moderada a severa. Para esta área de manejo de pacientes COVID-19 se contó con la participación de los residentes de posgrado de todas las especialidades del hospital. Aunque solo un tercio de los encuestados refirió haber asistido a esta área de aislamiento COVID del hospital, más de la mitad del personal de cirugía tuvo contacto con pacientes quirúrgicos sospechosos de COVID-19 ya sea durante la cirugía, en el preoperatorio y/o en el posoperatorio, confirmando así la necesidad del uso de EPP durante las cirugías y la evaluación de los pacientes en el área de emergencia de cirugía.

La mayoría de los participantes refirió no haber presentado síntomas, sin embargo, se le realizó prueba de hisopado nasofaríngeo a más de la mitad de los participantes resultando positivo más de la mitad de estos. Un número significativo de encuestados no conocía el resultado de su prueba lo cual refleja el preocupante retraso que presentan estas pruebas al estar centralizadas por las autoridades en materia de salud. Estos médicos pudieron haber estado contagiados y no cursar con sintomatología. ${ }^{(6)}$

Afortunadamente, la mayoría de los participantes presentó sintomas leves, ningún participante refirió haber presentado síntomas severos ni haber requerido hospitalización. Todos los casos sintomáticos fueron enviados a aislamiento domiciliario hasta cumplir con los protocolos de reincorporación establecidos por la Organización Mundial de la Salud y aplicados en nuestro hospital. ${ }^{(7)}$

El personal que resultó tener PCR positivo en su mayoría refirió haberse contagiado en el hospital, logrando identificar el origen de su infección o haber tenido contacto con alguna persona sintomática sin la protección pertinente, lo cual nos indica nuevamente que aumentar las medidas de protección necesariamente debe llevar a una disminución del porcentaje de contagios

Es llamativo el hecho de que la mitad de los encuestados refirió como causa de su contagio el contacto con un personal de salud que posteriormente resultó positivo para COVID-19. Múltiples factores pueden explicar este hecho, todos en relación al contacto entre personal de salud en áreas del hospital distintas a la emergencia, el quirófano o las salas de hospitalización. Las residencias, comedores, dormitorios, etc. pueden ser sitios de abandono de las medidas de protección, lo cual pudiera sugerir que la protección debería extenderse hasta estas áreas y no limitarse a los espacios habituales de trabajo, siendo necesario establecer protocolos para el manejo del personal de salud con sus compañeros

Otra variable importante es el uso de los EPP. Menos de la mitad de los participantes refirieron utilizar siempre el equipo de protección personal, mientras un porcentaje nada despreciable refirió no haber utilizado nunca equipos de protección personal. En el mismo orden de ideas, algunos participantes indicaron no conocer y/o no aplicar los protocolos para colocar y retirar los EPP. Estos resultados son similares a los publicados por la Federación Médica Colombiana quienes reportan que solo el 12\% de los encuestados usa completo los EPP (8), al igual que otros estudios que reflejan la dimensión de problemas vinculados con la falta de equipos de protección en el personal de salud. (9) 
Aunque la mayoría de los participantes refirió la utilización del gel alcoholado se debe promover y reforzar el cumplimiento de esta medida de higiene de manos en todo el personal de salud, tomando en consideración la recomendación del Centro de Control y Prevención de Enfermedades (CDC) acerca del uso de desinfectantes a base de alcohol al 60-95\%, en la mayoría de las situaciones clínicas, o del lavado de manos con agua y jabón, en aquellos casos en los cuales las manos estén visiblemente sucias, esto debido a que se evidencia un mayor cumplimiento en comparación al agua y el jabón ya que generalmente son menos irritantes y no ameritan lavamanos. ${ }^{(10)}$

Por último es importante resaltar el hecho de que no se están utilizando los EPP recomendados según el nivel de atención, de acuerdo a los protocolos establecidos por el CDC. (10) Se pudo evidenciar el uso incompleto del EPP por algunos de los participantes en procedimientos generadores de aerosoles, lo cual nos llevaría a investigar si el uso incompleto del mismo es por falta de información de los participantes o por no poseer los mismos.

El aumento de casos en la población general y personal de salud de COVID-19 debe alertar a nuestros servicios de cirugía general acerca de la necesidad de tomar medidas de protección de forma precoz para así garantizar la seguridad de los pacientes y de los cirujanos, ameritando modificar la manera como enfrentamos las actividades quirúrgicas y asistenciales. Se deben establecer y cumplir protocolos en cuanto al manejo del paciente en el área de la emergencia, quirófano y áreas de hospitalización, disminuir el número de acompañantes y mejorar el interrogatorio de sintomatología respiratoria para así tomar las medidas necesarias. (11)

Evitar las reuniones y mantener el uso de mascarilla en todo momento mientras se encuentre en el centro de salud, incluso en salas de descanso u otros espacios donde puedan encontrarse compañeros de trabajo son medidas sencillas de aplicar y que probablemente disminuirá el riesgo de contagio.

Mejorar el uso de los EPP según el nivel de atención, educar al personal de salud sobre el procedimiento para aplicar y retirar el mismo, tomar en consideración la responsabilidad individual de protegerse y proteger a nuestros pacientes, así como demandar de nuestras instituciones la responsabilidad de brindar los EPP, debe ser nuestra principal tarea como miembros del personal de salud de nuestro país.

\section{CONFLICTO DE INTERESES}

Los autores declaran no tener conflicto de intereses.

\section{REFERENCIAS}

1. Wei-jie Guan, Zheng-yi Ni, Yu Hu, Wen-hua Liang, Chun-quan Ou, Jian-xing He y colaboradores. Clinical Characteristics of Coronavirus Disease 2019 in China. N Engl J Med 2020; 382:1708-1720.

2. Alvarez M, Gortazar A, Pascual I, Rubio I, Barragan C, Alvarez E, Diaz J. Impacto de la pandemia por SARS-CoV-2 sobre la actividad y profesionales de un servicio de Cirugía General y del Aparato Digestivo en un hospital terciario. Cirugía Española. Elsevier. España 202; $98(6): 320-327$.

3. Gaceta Oficial Extraordinaria N6.519 de fecha 13 de marzo de 2020, fue publicado el Decreto N 4.160.

4. Adams J, Walls Ron. Supporting the Health Care Workforce During the COVID-19 Global Epidemic. JAMA 2020;323(15)

5. RD Algieri. Trauma, emergencias, en tiempos de COVID-19. Rev Argent Cirugía 2020;1 12(3):222-224.

6. Hanalise V Huff, Avantika Singh, Asymptomatic Transmission During the Coronavirus Disease 2019 Pandemic and Implications for Public Health Strategies. Clinical Infectious Diseases. Oxford academic. Mayo 2020. Disponible en: https://doi.org/10.1093/cid/ciaa654.

7. Organización Mundial de la Salud. Criterios para poner fin al aislamiento de los pacientes COVID 19. Reseña Científica. 17 junio 2020 Disponible en: https://apps.who.int/iris/handle/ 10665/332451.

8. Federación Médica Colombiana. Encuesta de reporte de exposición del personal de salud en el contexto de la pandemia SARS COV2 COVID-19 y condiciones de bioseguridad. Bogotá Mayo 2020. Disponible en: https://www.federacionmedicacolombiana.com/ wp-content/uploads/2020/05/05-2020-INFORME-SEGUNDAENCUESTA-FMC-CMB-COVID 19.pdf.

9. Ortiz Z, Antonetti L, Capriati A, Ramos S, Romeros M, Mariani J y colaboradores. Preocupaciones y demandas frente a COVID-19. Encuesta al personal de salud. ISSN 1669-9 106. Buenos Aires 2020;80

10. Hand Hygiene Recommendations. Guidance for Healthcare Providers about Hand Hygiene and COVID-19. National Center for Immunization and Respiratory Diseases (NCIRD), Division of Viral Diseases. Mayo 2020.

11. Ramos A, Anton R, Arribalzaga E, Sarotto L. COVID-19. Planificación del área quirúrgica. Rev Argent Cirugía 2020 - http://dx.doi. org/10.25132/raac.v1 12.n3.1527.es. 